\title{
Modulation of Gut Microbiota to Enhance Effect of Checkpoint Inhibitor Immunotherapy
}

\author{
Jianmin $W u^{1}$, Shan $W a n g^{2}$, Bo Zheng ${ }^{3}$, Xinyao Qiu ${ }^{2}$, Hongyang Wang ${ }^{1,3^{*}}$ and Lei Chen ${ }^{3 *}$ \\ 1 Institute of Metabolism \& Integrative Biology (IMIB), Fudan University, Shanghai, China, ${ }^{2}$ Department of Oncology, \\ Shanghai Medical College, Fudan University Shanghai Cancer Center, Fudan University, Shanghai, China, ${ }^{3}$ The International \\ Cooperation Laboratory on Signal Transduction, Eastern Hepatobiliary Surgery Hospital, Second Military Medical University, \\ Shanghai, China
}

OPEN ACCESS

Edited by:

Alexandr Bazhin,

LMU Munich University Hospital, Germany

Reviewed by: Ming Yi,

Huazhong University of Science and

Technology, China

Chiao-En Wu,

Linkou Chang Gung Memorial Hospital, Taiwan

Meriem Messaoudene,

University of Montreal Hospital Centre

(CRCHUM), Canada

*Correspondence:

Hongyang Wang

hywangk@vip.sina.com

Lei Chen

Chenlei@smmu.edu.cn

Specialty section:

This article was submitted to

Cancer Immunity

and Immunotherapy,

a section of the journal

Frontiers in Immunology

Received: 18 February 2021 Accepted: 14 June 2021

Published: 29 June 2021

Citation:

Wu J, Wang S, Zheng B, Qiu X, Wang $H$ and Chen L (2021)

Modulation of Gut Microbiota to

Enhance Effect of Checkpoint Inhibitor Immunotherapy.

Front. Immunol. 12:669150. doi: 10.3389/fimmu.2021.669150
Accumulating evidence demonstrated the crucial role of gut microbiota in many human diseases, including cancer. Checkpoint inhibitor therapy has emerged as a novel treatment and has been clinically accepted as a major therapeutic strategy for cancer. Gut microbiota is related to cancer and the effect of immune checkpoint inhibitors (ICls), and supplement with specific bacterial species can restore or enhance the responses to the ICls. Namely, specified bacteria can serve as the biomarkers for distinguishing the patient who will respond to ICls and determine the effectiveness of ICls, as well as predicting the efficacy of checkpoint inhibitor immunotherapy. Regardless of the significant findings, the relationship between gut microbiota and the effect of $\mathrm{ICls}$ treatment needs a more thorough understanding to provide more effective therapeutic plans and reduce treatment complication. In this review, we summarized the role of gut microbiota played in immune system and cancer. We mainly focus on the relationship between gut microbiota and the checkpoint inhibitor immunotherapy.

Keywords: cancer treatment, tumor, immune checkpoint inhibitor, tumor immunotherapy, PD-1, CTLA-4

\section{INTRODUCTION}

Microbes have been on earth for billions of years, they nearly occupy every corner of the earth, including the human body. The majority of microbes in our body resides in the gut (1). Gut microbiota consists of bacteria, fungi, virus, and other microbial and eukaryotic species and they mainly located in small intestinal and colon $(1,2)$. Gut microbiota colonized our body when we were born (3), and it has a great variety between different individuals (4). Based on previous research, gut microbiota has a close relation with various pathological conditions, including obesity (5), diabetes (6), neurodegenerative diseases (7), and cancers (8-11). Cancer is a major threat to human's health with high fatality rate. At present, the major therapeutic strategy of cancer includes surgery, radiotherapy, chemotherapy, and immunotherapy (12). Cancer immunotherapy is a new therapeutic strategy emerging in recent years. Of note, cancer immunotherapy targeting immune checkpoints has achieved a great success. Antibody drugs targeting cytotoxic T-lymphocyteassociated protein 4 (CTLA-4) and programmed cell death protein 1 (PD-1) or its ligands programmed cell death protein ligand 1 (PD-L1) and 2 (PD-L2) arise in these years (13). The relevant antibody drugs like nivolumab, pembrolizumab were approved in clinical application by 
the United States Food and Drug Administration (FDA) (14). Most patients receiving ICIs treatment can achieve long-term suppression (13). But there still exist some limitations of ICIs, the response rates of patients treated with anti-PD-1 antibody are relatively low in several types of cancer (approximately 20 to 25\% in non-small-cell lung cancer, melanoma, or renal-cell cancer) (15). What's more, small number of patients gained hyperprogression after treating with ICIs (16), and approximately one third of patients relapse after treating with ICIs (13).

Recent studies reveal that the abundance and composition of gut microbiota can influence the effect of ICIs treatment (17). The relevant bacteria such as Bifidobacterium (18), Bacteroides fragilis (19), Akkermansia muciniphia (20) are found to be related to the clinical outcomes of cancer immunotherapy. Gut microbiota might serve as a potential factor affecting the effectiveness of checkpoint blockade immunotherapy. In this review, we summarized the role that gut microbiota played in immune system and in cancer genesis and development, we mainly focus on the relationship between gut microbiota and the effect of ICIs treatment.

\section{GUT MICROBIOTA AND IMMUNE SYSTEM}

\section{Gut Microbiota and Innate Immunity}

The innate immune system has physiological functions protecting our body. The innate immune system in intestinal consists of epithelial cells, myeloid cells, innate lymphoid cells, and other types of cells (21). Gut microbiota has broader effects on innate immunity (22). The immune system inside the intestinal plays a vital role in preventing external bacterial invasion and infection (22). Gut mucus is the first barrier providing the underlying epithelium from bacteria, to some extent, gut mucus is shaped by microbiota. The penetrability of gut mucus of germ-free mice and conventionally raised mice is different, the small intestine mucus become normally detached and colonic inner mucus become impenetrable after gavaged with microbiota from conventionally raised mice (23). In addition, immunoglobulin $\mathrm{A}(\operatorname{IgA})$ is an antibody isotype existing in intestinal lumen (24), mucosal IgA is binding to the polymeric immunoglobulin receptor and secreted across the epithelium (25), and it has a close relationship with Bacteroides fragilis. A research found that Bacteroides fragilis can take advantage of the immune system to settle down in the intestine of mice (Figure 1), however, it is difficult for Bacteroides fragilis to settle on the surface of the intestine and maintain long-term stability once the mice lack $\operatorname{IgA}$, indicating that the immune system has a close relationship with commensal bacteria (26).

\section{Gut Microbiota and Adaptive Immunity}

Gut microbiota has a close relation with adaptive immunity, especially $\mathrm{T}$ cells. A research found that in mice lamina propria, Th17 cells can be induced by Segment filamentous bacteria (SFB). Transplantation of SFB in mice induces the appearance of CD4+
T helper cells, increases level of IL-17 and IL-22 in lamina propria (Figure 1) (27). This research reveals that certain bacteria can cause changes in the number of specific cells in the immune system and expression of immune-related genes in vitro. However, how SFB affects Th17 cells remains unclear. Another research found that Indigenous Clostridium species promote the accumulation of CD4+ T regulatory cells (Tregs) in colonic mucosa. They found clusters IV and XIVa of the genus Clostridium is associated with Tregs accumulation in the colon (Figure 1) (28). Tregs plays an important role in maintenance of immune homeostasis, so this finding might offer a new therapeutic method to autoimmunity disease and allergy (28, 29). Gut microbiota influences immune system mainly through metabolites such as short-chain fatty acids (SCFAs). SCFAs are considered as the most abundant metabolites produced by gut microbiota in gut lumen, and they are produced by various types of bacteria including Faecalibacterium prausnitzii, Roseburia intestinalis, and Anaerostipes butyraticus (30, 31). SCFAs consist of acetate, propionate, and butyrate, they have an extensive influence on immune system. Studies have shown that butyrate can induce Treg cell differentiation in vivo or in vitro (32). Further research found that butyrate regulates Foxp3, which demonstrates that butyrate plays a role in inhibiting the activation and proliferation of $\mathrm{T}$ cells $(32,33)$. Butyrate also has an impact on Th17 cells through controlling the cytokine production by dentritic cells (DCs) (Figure 1). Butyrate produced by gut microbiota increases the level of GPR109a and induces expressing of IL-10 and Aldh1a1 in colonic macrophage and DCs, result in suppressing of development of Th17 cells (34). Feeding mice with Bifidobacterium infantis increased the production of retinoic acid in dendritic cells (DCs), result in increasing of TGF- $\beta$ production and Treg expansion (35). Studies with gnotobiotic mice reveals an immunomodulatory effect of secreted microbial metabolites. In mice, Bacteroides fragilis secreted polysaccharide A binding to Toll-like receptor 2 (TLR2) on Treg cells, which can enhance the expansion of Tregs while suppressing the proinflammatory Th17 response (Figure 1), this indicated that polysaccharide A secreted by Bacteroides fragilis is able to prevent and cure experimental colitis in animals $(36,37)$. Through reading all of these researches, we can conclude that gut microbiota is indispensable for maintaining the immune homeostasis in animals and in human.

\section{GUT MICROBIOTA AND CANCER}

\section{Gut Microbiota and Gastrointestinal Cancer}

Gut microbiota has a great influence on gastrointestinal cancer $(38,39)$. The colonization of Helicobacter pylori (H. pylori) causes a persistent inflammatory response, which can lead to cancers of the stomach (38). Infected individuals with low abundance of $H$. pylori have a lower risk of gastric cancer, proving that $H$. pylori is associated with gastric carcinogenesis (40). Further studies revealed that $H$. pylori might promote 


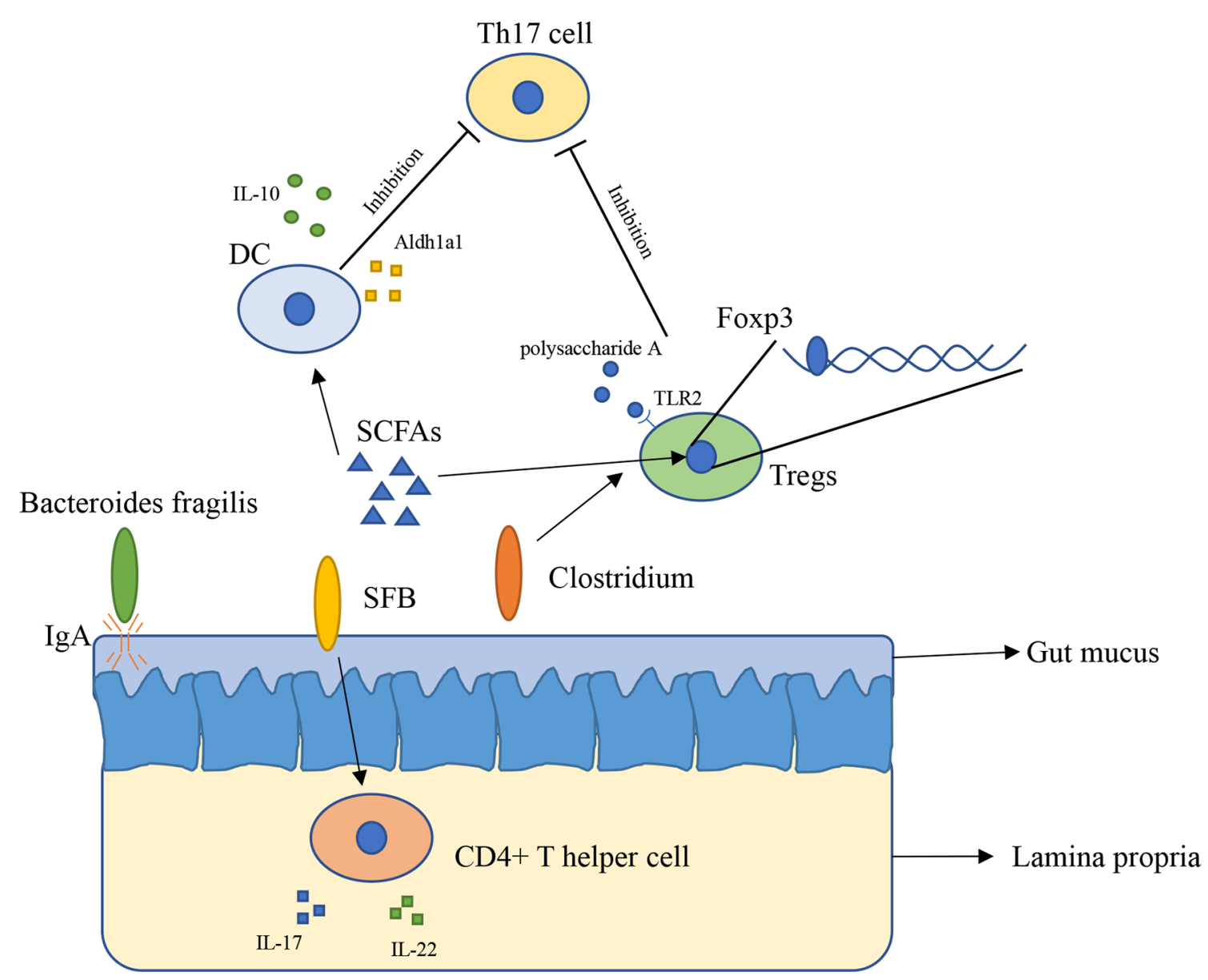

FIGURE 1 | The interaction between gut microbiota and immune system (1). Bacteroides fragilis takes advantage of IgA to settle down in the intestine of mice (2); SFB induces the appearance of CD4+ T helper cells in lamina propria (3); Clusters IV and XIVa of the genus Clostridium are associated with Tregs accumulation in colon (4); SCFAs regulate Foxp3 in Tregs, inhibit immune response of T cells (5); SCFAs induce expressing of IL-10 and Aldh1a1 in DCs, result in inhibiting the development of Th17 cell (6); Polysaccharide A secreted by Bacteroides fragilis binds to TLR2 in Tregs, enhances Tregs and suppresses the proinflammatory Th17 response.

cancer through $\beta$-catenin signal pathway (38). Colorectal cancer (CRC) is associated with specific bacteria including Bacteroides fragilis, Fusobacterium nucleatum, Porphyromonas asaccharolytica, Parvimonas micra, Prevotella intermedia, Alistipes finegoldii, and Thermanaerovibrio acidaminovorans, which can serve as potential diagnostic markers across population (41). Certain types of gut microbes might promote CRC. Sunny H. Wong et al. feed conventional and germ-free mice with stool samples from CRC patients and healthy individuals, then they found mice feeding with stool samples from CRC patients developed high-grade dysplasia $(\mathrm{P}<.05)$ and macroscopic polyps $(\mathrm{P}<.01)$ than mice feeding with stool samples from healthy individuals (42). Shaoguang W et al. found that Enterotoxigenic Bacteroides fragilis (ETBF) promotes colon tumorigenesis by stimulating immune response via Th17 cells in mouse CRC model, ETBF stimulates rapid colitis and colon tumors in multiple intestinal neoplasia (Min) mice and induce activation of transcription-3 (Stat3) in the colon of Min mice, Stat3 signaling is necessary for the generation of TH17 cells, and IL-17 produced by CD4+ Th17 is sufficient to induce tumorigenesis in the absence of $\gamma \delta$ T cells (43). Gut microbiota also has a direct role in the occurrence of oncogenic mutations in colorectal cancer, a research reveals that colibactin-a compound generated by Escherichia coli-is believed to alkylate DNA on adenine residues and cause DNA double-strand breaks, which is related to the generation of colorectal cancer (44). Tumor promoting effects are mostly related to depletion or dysbiosis of gut-microbiota. After killing some bacteria with antibiotics (ATBs) in mice, the development of the tumor in the liver and the colon can be reduced $(45,46)$. Dianne $H$. Dapito et al. feed mice with ATBs which can eliminates commensal bacteria and reduce systemic lipopolysaccharide levels, then they can be found in DEN plus CCl4 HCC model, the tumor size was reduced (45).

\section{Gut Microbiota and Liver Cancer}

Liver does not have direct contact with microbiota, but it has tight anatomic links to the gut $(47,48)$. In most of the studies, liver 
cirrhosis is a major potential risk of liver cancer (49). In 2016, people noticed that gut microbiota might associate with HCC progression in cirrhotic patients (50). Until now, there is no clinical evidence showing that a specific bacteria is associated with HCC (51). But experiments on gut-sterilized mice or germfree mice showed that the development of HCC might associate with microbiota or microbially activated pathways, microbial metabolites, or microbiota-associated molecular patterns (MAMPs) also associate with the development of HCC (51). Gut microbiota and Toll-like receptors (TLRs) are required for HCC promotion but not required for HCC initiation (45). One research revealed that gut microbiota is associated with cholangiocarcinoma. According to the study which included 60 patients with cholangiocarcinoma, there existed a distinct, tissue-specific microbiome in bile duct tissues. Compared with normal samples (non-neoplastic liver), the patients with cholangiocarcinoma (CCA) tumors had a significant increase in Stenotrophomonas species in bile duct tissues (52).

\section{Gut Microbiota and Other Types of Cancer}

There are also several studies focused on the gut microbiota and breast cancer. In 2015, Bard and his colleagues observed that gut microbiota was associated with clinical stages of breast cancer. They collected fecal samples from different stages of breast cancer patients, and performed 16S rRNA sequencing, they found that stage I patients had a lower absolute number of Blautia sp. than stage III, several other bacterial species (Bifidobacterium, Blautia, F. Prausnitzii, and Blautia) were different according to different clinical stages of breast cancer (53). Another research also found that gut microbiota was associated with different stages of breast cancer, compared to patients in stage $0 / \mathrm{I}$, the number of Bacteroidetes, Clostridium coccoides cluster, Clostridium leptum cluster, Faecalibacterium prausnitzii, and Blautia sp. are increased in stage II/III patients. In addition, the absolute numbers of total bacteria are different according to the patient's body mass index, which illustrated that obesity is associated with gut microbiota (54). Gut microbiota and lung has an essential cross-talk called "gut-lung axis" (55). In lung cancer patients, the level of Enterococcus sp. was decreased and the levels of Actinobacteria sp. and Bifidobacterium sp. were increased (56), indicating that lung cancer had an impact on the composition of gut microbiota.

\section{Metabolite of Gut Microbiota and Cancer}

Metabolites of gut microbiota are known to induce proinflammatory cytokines and mediate tumor-associated inflammation in colon cancer $(57,58)$. The short chain fatty acids (SCFAs) secreted by bacteria (especially propionic acid and butyric acid) can mediate anti-inflammatory response and reduce the occurrence of colorectal cancer (59). Besides, SCFAs mediate 21 gene expression through modulation of microRNA, which will in turn influence colonic carcinogenesis (60). Bacteroides fragilis in the intestines of patients with colorectal cancer produce a variety of toxic metabolites such as $\beta$ glucuronidase, spermine oxidase, reactive oxygen species, reactive nitrogen species, and nitroso compounds, which can induce DNA damage and promote colorectal cancer $(61,62)$. Bile acid is an important component synthesized by the liver to regulate fat metabolism. Some anaerobic bacteria in intestine such as Bacteroides metabolize bile acids into secondary bile acids, which can cause DNA damage, stimulate EGFR or wnt/ $\beta$ cantenin pathway, inducing colorectal cancer or liver cancer (46, $63,64)$. Dietary or genetic obesity induces alterations of gut microbiota. Fed mice with high-fat diet resulting in the increase of Gram-positive bacteria (46). High-fat diet also increases the levels of bacterially generated deoxycholic acid (DCA). DCA is an intestinal bacterial metabolite which can cause DNA damage (65). Accumulating of DCA can facilitate obesity-associated HCC development in mice (46). In liver cancer, bile acid metabolism changes caused by gut microbiota regulates the expression of CXCL16 and recruits CXCL16-mediated natural killer T (NKT) cells, which could control the liver tumor growth (66). NKT cells can kill tumor cells in a CD1d-dependent manner (51). Recent study revealed that inosine-a purine nucleoside molecule produced by gut microbiota-can enhance cancer immunotherapy response in mice (67).

\section{GUT MICROBIOTA IS ASSOCIATED WITH THE EFFECT OF ICI IMMUNOTHERAPY}

\section{Bifidobacterium}

In 2015, Thomas and his colleagues first noticed that there were correlations between gut microbiota and ICI immunotherapy (18). They used mice which were harbored with different commensal microbiota, then compared the melanoma growth of these mice. They also found that different microbiota might relate to different spontaneous antitumor immunity. Of which, they found that Bifidobacterium could facilitate antitumor effect of PD-L1 blockade (Table 1). Commensal Bifidobacterium controlled the growth of melanoma tumor in mice, oral administration of Bifidobacterium was an effective way to block tumor growth. Bifidobacterium treated mice showed a better antitumor effect compared to non-Bifidobacterium treated mice and this tumor control effect was related to tumor-specific $\mathrm{T}$ cells in periphery and accumulation of antigen-specific CD8 $+\mathrm{T}$ cells within the tumor (18). This study demonstrated that commensal Bifidobacterium can enhance antitumor immunity in vivo with an antigenindependent manner and it has a synergistic effect with PD-L1 blockade (18). In 2020, another team found that Bifidobacterium enhanced antitumor immunity through production of metabolite inosine (68). In this research, they found three bacterial species-Bifidobacterium pseudolongum, Lactobacillus johnsonii, and Olsenella species, these three bacterial species can significantly enhance the efficacy of ICIs in mouse models of CRC (Table 1) (68). They found that Bifidobacterium pseudolongum can enhance the antitumor effect through metabolite inosine, and this effect was dependent on adenosine $2 \mathrm{~A}$ receptor $\left(\mathrm{A}_{2 \mathrm{~A}} \mathrm{R}\right)$ signaling specifically in $\mathrm{T}$ cells. They also found that the antitumor effect of Akkermansia muciniphia in human also relies on inosine- $\mathrm{A}_{2 \mathrm{~A}} \mathrm{R}$ signal (Figure 2). Recent research found that Bifidobacterium can enhance local anti-CD47 immunotherapy in tumor (71). The 
TABLE 1 | Modulatory function of gut microbiome in ICls therapy.

\begin{tabular}{|c|c|c|c|c|c|c|}
\hline Bacteria & Model & Treatment & Cancer & Modulatory function in ICI therapy & Author/Year & Ref \\
\hline Bifidobacterium & Mouse & PD-L1 blockade & Melanoma & $\begin{array}{l}\text { a) Improve antitumor immunity } \\
\text { b) Enhancing dendritic } \\
\text { cell function } \\
\text { c) Enhancing local anti-CD47 } \\
\text { immunotherapy in tumor }\end{array}$ & Ayelet Sivan 2015 & $(18)$ \\
\hline $\begin{array}{l}\text { Bifidobacterium } \\
\text { pseudolongum } \\
\text { Lactobacillus johnsonii } \\
\text { Olsenella species }\end{array}$ & Mouse & $\begin{array}{l}\text { Immune } \\
\text { checkpoint } \\
\text { blockade }\end{array}$ & $\mathrm{CRC}$ & $\begin{array}{l}\text { Increasing metabolite inosine } \\
\text { production }\end{array}$ & Lukas F. Mager 2020 & $(68)$ \\
\hline Akkermansia muciniphia & $\begin{array}{l}\text { Human/ } \\
\text { Mouse }\end{array}$ & PD-1 blockade & $\begin{array}{l}\text { Epithelial } \\
\text { tumors }\end{array}$ & $\begin{array}{l}\text { Enhancing the antitumor effect of PD-1 } \\
\text { blockade }\end{array}$ & Bertrand Routy 2018 & $(20)$ \\
\hline $\begin{array}{l}\text { Bacteroides fragilis } \\
\text { Bacteroides thetaiotaomicron } \\
\text { Burkholderiales }\end{array}$ & $\begin{array}{l}\text { Human/ } \\
\text { Mouse }\end{array}$ & $\begin{array}{l}\text { CTLA-4 } \\
\text { blockade }\end{array}$ & $\begin{array}{l}\text { Melanoma/ } \\
\text { colon cancer }\end{array}$ & $\begin{array}{l}\text { Influence interleukin } 12 \text { (IL-12)- } \\
\text { dependent } \mathrm{TH} 1 \text { immune responses }\end{array}$ & Marie Vétizou 2015 & (19) \\
\hline $\begin{array}{l}\text { Uminococcaceae family } \\
\text { Clostridiales order } \\
\text { Faecalibacterium genus }\end{array}$ & $\begin{array}{l}\text { Human/ } \\
\text { Mouse }\end{array}$ & PD-1 blockade & Melanoma & $\begin{array}{l}\text { Promote the infiltration of CD } 8+T \text { cells } \\
\text { in tumors }\end{array}$ & Gopalakrishnan V 2018 & $(17)$ \\
\hline Ratio of Prevotella and Bacteroides & Human & $\begin{array}{l}\text { PD-1/PD-L1 } \\
\text { blockade }\end{array}$ & $\begin{array}{l}\text { Gastrointestinal } \\
\text { cancer }\end{array}$ & $\begin{array}{l}\text { Related to nucleoside and nucleotide } \\
\text { biosynthesis, lipid biosynthesis, sugar } \\
\text { metabolism, and fermentation to } \\
\text { short-chain fatty acids }\end{array}$ & Zhi Peng 2020 & (69) \\
\hline $\begin{array}{l}\text { Bifidobacterium longum, Collinsella } \\
\text { aerofaciens, and Enterococcus faecium }\end{array}$ & Human & PD-L1 blockade & Melanoma & $\begin{array}{l}\text { a) Decreasing regulatory } T \text { cell } \\
\text { b) Increasing Batf3 dendritic cells } \\
\text { c) Enhancing Th1 responses }\end{array}$ & Vyara Matson 2018 & $(70)$ \\
\hline
\end{tabular}

researchers used wild type (WT) mice, ATB-fed mice, and sterile mice to test the antitumor effects of CD47-based immunotherapy. The result showed that intestinal microbes outside the gastrointestinal tract are effective in promoting CD47 tumor immunotherapy. The results of Bifidobacterium injection experiments show that Bifidobacterium has antitumor effects in mice that do not respond to CD47 inhibition (71). This study reveals that the tumor-targeting ability of Bifidobacterium might be a possible mechanism that gut microbiota affect the antitumor response.

\section{Bacteroide}

In 2015, another group published a research article focused on the gut microbiota and the treatment of CTLA-4 blockade (19). They find that the effect of CTLA-4 blockade treatment is related to distinct Bacteroides species (Bacteroides fragilis and/or Bacteroides thetaiotaomicron and Burkholderiales) (Table 1). If gut microbiota was removed or destroyed in mice, then CTLA-4 blockade has no effect on tumors. Transplant with $B$. fragilis can benefit for antitumor effect of CTLA-4 blockade. They found that Bacteroides thetaiotaomicron or Bacteroides fragilis cause $\mathrm{T}$ cell response, which is associated with antitumor efficacy of CTLA-4 blockade (19). Treatment of anti-CTLA-4 blockade also influence the abundance of immunogenic Bacteroides spp. in the gut, which would in turn affect the antitumor efficacy of ICIs. Further research revealed that CTLA-4 blockade influences the composition of microbiota, oral feeding of Bacteroides fragilis induce Th1 immune response, promoting the maturation of dendritic cell, which facilitate tumor control in mice and patients (Figure 2) (19). However, a research focused on the PD-1 immunotherapy of melanoma patients revealed that responders have a lower abundance of Bacteroidales than the non-responders (17). They collected 112 melanoma patients who were undergoing anti-PD-1 immunotherapy, then they examined the gut microbiome of these patients, and they found the diversity and composition of gut microbiota are different between the responders and non-responders: Clostridiales order and Ruminococcaceae family are enriched in responders while Bacteroidales order are enriched in nonresponders. The abundance of Bacteroidales were positively related to the frequency of Treg cells and myeloid-derived suppressor cells (17). This study revealed that the microbiome composition might have an impact on anti-PD-1 immunotherapy, patients with favorable gut microbiome like Ruminococcaceae or Faecalibacterium might have an enhancing antitumor immune response, and this antitumor immune response was related to more antigen presentation in the periphery and improved effector $\mathrm{T}$ cell function in the tumor microenvironment. On the contrary, patients with a high abundance of Bacteroidales might have a limited response to anti-PD-1 immunotherapy (17).

\section{Akkermansia muciniphila}

PD-1-based immunotherapy is also associated with gut microbiota. Analyzing of stool samples from renal cell carcinoma (RCC) and non-small cell lung cancer (NSCLC) patients after treating with PD-1 blockade revealed that clinical outcomes were associated with the abundance of Akkermansia muciniphia, oral supplementation with Akkermansia muciniphia to non-responders restored the efficacy of PD-1 blockade, which demonstrated that the Akkermansia muciniphia can enhance the effect of PD-1 inhibitor (Table 1) (20). They also found that treating with ATB in mice inhibited the antitumor effect of PD-1 blockade, which demonstrated that dysbiosis of gut microbiome 


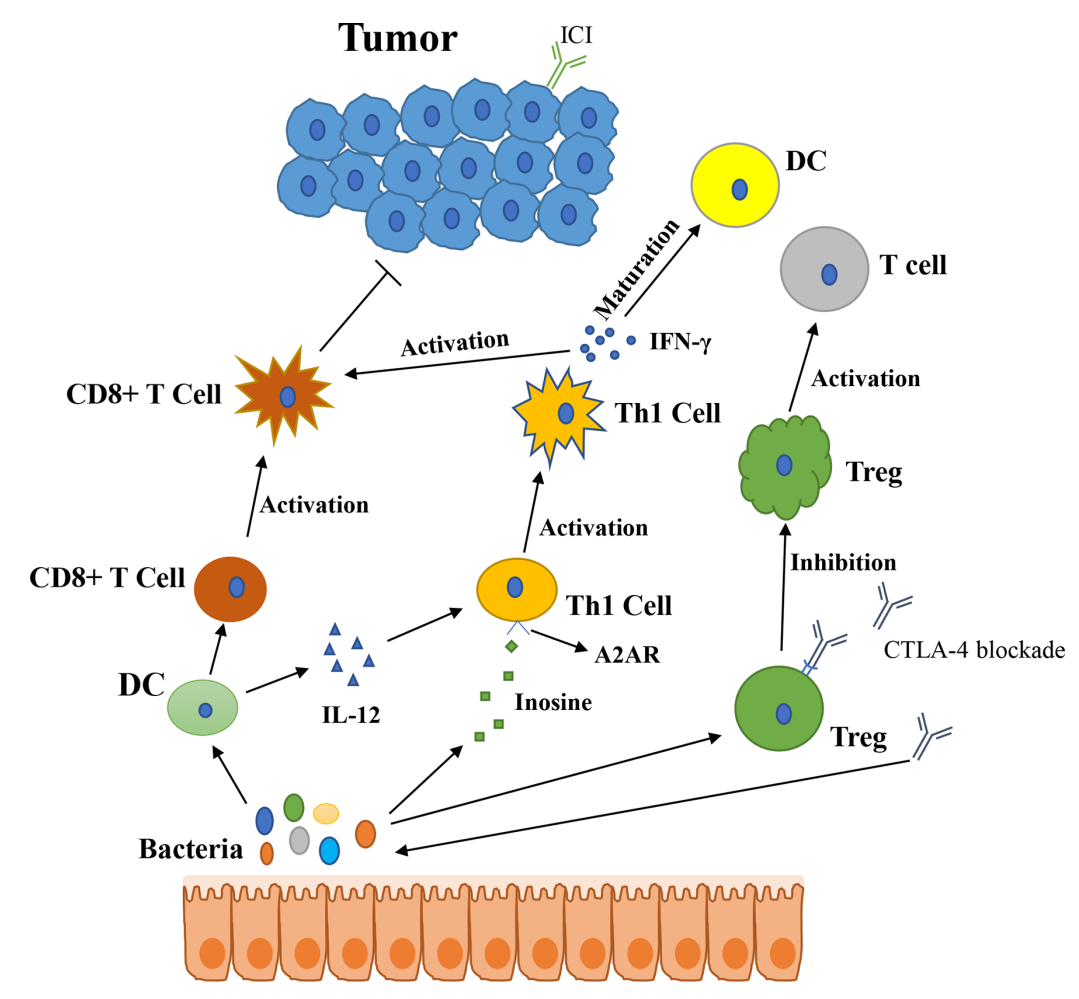

FIGURE 2 | The potential mechanism of gut microbiota modulating the efficacy of ICls (1). Bacteroides thetaiotaomicron or Bacteroides fragilis enhances T cell response, treatment of anti-CTLA-4 blockade influences the abundance of immunogenic Bacteroides spp., which will in turn enhance immune response of $T$ cells (2); Bacteroides fragilis induces Th1 immune response, promotes the maturation of dendritic cell (3); Bifidobacterium improves function of DC, results in activating of CD8+ T cell and enhancing anti-PD-L1 antitumor effect (4); The metabolite inosine of gut microbiota activates Th1 cell and increases level of IFN- $\gamma$, which can enhance antitumor effect in vivo (5); Microbiota composition affects IL-12-independent Th1 cell immune response (6); CTLA-4 blockade induces the inactivation of CTLA-4+ Treg cells, leads to activating of effector T cell and enhancing antitumor effect.

system is another factor affecting results of ICIs (20). Fecal microbiota transplantation (FMT) from patients who respond to ICIs to mice can enhance the antitumor effect of PD-1 blockades in mice, while FMT from non-responders failed to do so. Oral supplementation with Akkermansia muciniphia to non-responders can enhance the efficacy of PD-1 blockade, besides, a higher incidence of E. hirae was found in responders of NSCLC patients than in non-responders (20). Another research consisting of 31 metastatic renal cell carcinoma (mRCC) patients also found that the abundance of Akkermansia muciniphila is positively correlated with the outcomes of checkpoint inhibitors. After treating with nivolumab or nivolumab plus ipilimumab, stool samples were collected and analyzed from mRCC patients, the result revealed that several bacteria are related to clinical benefit. They also found that a better treatment outcome is associated with high microbial diversity (72). The mechanism of Akkermansia muciniphila which promotes the effect of ICIs might be related to inosine (Figure 2), Akkermansia muciniphila can produce inosine in vitro in ICIs-treated tumors. Anti-CTLA-4 immunotherapy combined with monocolonization of Akkermansia muciniphila increased antitumor immunity, and this was associated with $T$ cell expression of $A_{2 A} R$ (68). In HCC patients, Akkermansia muciniphila increased in responders after PD-1 immunotherapy. Besides, Ruminococcaceae spp. and other 18 species of bacteria were also increased in responders. In nonresponders, the level of Proteobacteria increased (73).

\section{Faecalibacterium, Clostridiales, and Ruminococcaceae}

Gopalakrishnan V et al. examined gut microbiome of melanoma patients who were treating with anti-PD-1 immunotherapy, they found the composition and diversity of responders are different from those of non-responders. Clostridiales order and Ruminococcaceae family and Faecalibacterium genus were enriched in responders and Bacteroidales order was enriched in non-responders (17). The existence of Ruminococcaceae family, Clostridiales order, and Faecalibacterium genus can promote the infiltration of CD8+ T cells in tumors (Table 1). Patients rich in Clostridiales, Faecalibacterium, or Ruminococcaceae in gut have a higher frequency of CD4+ and CD8+ T cells. Besides, patients with high abundance of Faecalibacterium had better antigen processing and presenting ability and a relatively high density of immune cells compared to patients with high abundance of Bacteroidales in the gut (17). 


\section{Prevotella/Bacteroides}

Since gut microbiota is a whole system, a small change in one species of bacteria may have an impact on others. A recent research reveals that after immunotherapy, the ratio of Prevotella and Bacteroides will be elevated (Table 1) (69). In a research which included 74 patients with advanced-stage gastrointestinal cancer, after anti-PD-1/PD-L1 treatment, they observed an elevation of Prevotella/Bacteroides ratio in patients with a preferred response to anti-PD-1/PD-L1 treatment and a particular responder harboring a significantly higher abundance of Prevotella, Ruminococcaceae, and Lachnospiraceae. Ruminococcaceae is also associated with a favorable objective response rate in NSCLC after treatment of immune checkpoint inhibitors (74). Besides, gut bacteria which are capable of SCFAs, were positively associated with anti-PD-1/PD-L1 response (69).

\section{Composition of Gut Microbiota}

As we discussed before, gut microbiota is a complex system. The alteration of whole system not just specific bacteria also plays an important role which can influence the effect of ICIs. Lisa Derosa et al. analyzed fecal samples from RCC patients treated with nivolumab, they found the patients who had used ATBs recently reduced objective response by $19 \%$ compared to the patients who had not used ATBs, and the composition of gut microbiota was significantly changed in these patients (75). This research indicates that ATBs shift the composition of gut microbiota, resulting in decreased efficacy of ICIs. Bertrand Routy et al. studied the impact of ATBs on NSCLC, RCC, and urothelial patients who were received anti PD-1/PD-L1 blockade, and they found that progression-free survival (PFS) and overall survival (OS) were significantly shorter in the ATB-treated group, demonstrating that dysbiosis has an impact on therapeutic efficacy of ICIs (20). Besides, alpha diversity is also important factor in the composition of gut microbiota. Gopalakrishnan V et al. analyzed fecal microbiome samples from 43 melanoma patients who were undergoing PD-1 blockade immunotherapy, and they found responding patients have a higher alpha diversity of bacteria than the non-responders, they also found that patients with higher alpha diversity in their fecal samples had significantly prolonged PFS than the patients in low alpha diversity (17).

\section{ONGOING CLINICAL TRIALS OF GUT MICROBIOTA IN ICls}

Using bacteria as a new method for cancer therapy aroused people's attention since bacteria were first applied in tumor therapy in 1991 (76). To date, there are three major ways in which the gut microbiota is used in tumor treatment: oral probiotics, diet intervention, and FMT. Several researches reported that probiotics have antitumor effects, especially in CRC. The antitumor effects of probiotics Clostridium butyricum and Bacillus subtilis on CRC progression had been proved in mice (77). A clinical research found that oral
Lactobacillus johnsonii can significantly reduce the tumor recurrence rate of colon cancer patients after surgery (78). Another 12-year prospective clinical study showed that longterm intake of high-dose yogurt (containing Streptococcus thermophilus and Lactobacillus bulgaricus) has a significantly reduced risk of colorectal cancer (79). However, although probiotics were proved to be beneficial with antitumor effects, probiotic supplementation with ICIs might generate conflicting results (80). Probiotic use after ATBs could have opposite effect and delay restoration of the gut microbiota (81). One study focused on melanoma patients reported that the effect of immunotherapy might be negatively influenced by probiotics (82). Thus, it is necessary to find out the relation and inner mechanism between probiotics and the effect of ICIs in other kind of tumors. Altered diet can rapidly change the composition of gut microbiome (83). Which means it might be a simple and safe way to modulate gut microbiome in ICI patients (84). Patients with advanced bowel cancer intake inulin rich in oligofructose can enhance the effect of chemotherapy (85). Some studies are ongoing to investigate the association of dietary intervention with ICIs (NCT03700437, NCT03595540).

To date, FMT had been widely used in many diseases including cancer treatment. Previous animal experiments showed that fed mice with specific bacteria or FMT can enhance the sensitivity of immunotherapy. Thus, make several specific bacteria into medicine might be a new clinical adjuvant treatment of immunotherapy to cancer. FMT had been already applied in clinical trials, Robert and his colleagues successfully treated immune checkpoint inhibitors-associated colitis using FMT, transplant of fecal microbiota reconstruct the gut microbiome of patients and increase the proportion of regulatory $\mathrm{T}$-cells in the colonic mucosa. This trial demonstrated that reshaping of gut microbiome might abrogate ICI-associated colitis (86). Recently, there were two studies using FMT to promote response in ICI-refractory patients $(87,88)$. A phase I clinical trial (NCT03353402) was performed in patients with anti-PD-1 refractory metastatic melanoma, in these patients, 3 of 10 patients were responded after intervention of FMT (88). Another research also focused on anti-PD-1 therapy in advanced melanoma, and they found that FMT and anti-PD-1 changed tumor microenvironment and gut microbiome which led to overcoming resistance of anti-PD-1 immunotherapy (87). In consideration of the relationship between the gut microbiota and ICIs, formulating a personalized treatment plan for patients based on the characteristics of the gut microbiota may become a new plan to optimize ICIs in tumor. Until now, several clinical trials are proceeding (Table 2). Most of clinical trials focused on the FMT combined with ICIs (NCT04163289, NCT04130763, NCT04130763, NCT04056026, NCT03341143, NCT04116775), a phase I trial (NCT03686202) using a new treatment approach named microbial ecosystem therapeutics (MET), which can also achieve FMT.

Several trials (NCT04208958, NCT03341143, NCT04116775) had preferable primary outcomes and moved to phase II trial. All of these clinical trials indicate that gut microbiota might be an auxiliary method for ICIs antitumor therapy. 
NCT03700437 Non-small Cell Lung Cancer

NCT03595540 Cancer

Breast Cancer

$$
\text { Colorectal Cancer }
$$

NCT03686202 All solid tumors

NCT04163289 RCC

NCT04130763 Gastrointestinal system cancer

NCT03353402 Melanoma

NCT04056026 Metastatic mesothelioma

NCT04208958 Metastatic cancer, melanoma, gastroesophageal junction

adenocarcinoma, colorectal cancer

NCT03341143 Melanoma

NCT04116775 Prostate cancer

NCT04136470 NSCLC, melanoma

NCT04169867 Melanoma

NCT04291755 NSCLC, colorectal cancer

NCT03353402 Melanoma

Pembrolizumab

Dietary intervention

Fast-Mimicking Diet

(FMD)

Opdivo, Keytruda

FMD for 5 days

sting-Mimicking Diet with Chemo-immunotherapy in Non-small

Cell Lung Cancer (NSCLC)

Fasting-Mimicking Diet in Patients Undergoing Active Cancer

Treatment

PD-1/PD-L1 blockade MET-4

Nivolumab, Ipilimumab FMT

PD-1 blockade

FMT

PD-1 blockade

FMT

Pembrolizumab

FMT

PD-1 blockade

Pembrolizumab

Pembrolizumab

\section{VE800, nivolumab,}

vancomycin ora

capsule

Nivolumab, Ipilimumab, -

Atezolizumab

Nivolumab, Ipilimumab, -

Atezolizumab

Pembrolizumab

\section{Pembrolizumab}

injection

PD-1 blockade
Feasibility Study of Microbial Ecosystem Therapeutics (MET-4) to Evaluate Effects of Fecal Microbiome in Patients on ImmunOtherapy (MET4-IO)

Preventing Toxicity in Renal Cancer Patients Treated With Immunotherapy Using Fecal Microbiota Transplantation (PERFORM)

Fecal Microbiota Transplant (FMT) Capsule for Improving the Efficacy of Anti- PD-1

Fecal Microbiota Transplantation (FMT) in Metastatic Melanoma Patients Who Failed Immunotherapy

A Single Dose FMT Infusion as an Adjunct to Keytruda for Metastatic Mesothelioma

Study of VE800 and Nivolumab in Patients With Selected Types of

Advanced or Metastatic Cancer (Consortium-IO)

Fecal Microbiota Transplant (FMT) in Melanoma Patients

Fecal Microbiota Transplant and Pembrolizumab for Men With

Metastatic Castration Resistant Prostate Cancer

BioForte Technology for in Silico Identification of Candidates for a $130 \quad$ Poland Discovery

New Microbiome-based Therapeutics and Diagnostics

Polish Microbiome Map

Development and Analysis of a Stool Bank for Cancer Patients

Fecal Microbiota Transplantation (FMT) in Metastatic Melanoma Patients Who Failed Immunotherapy

MET4, Microbial Ecosystem Therapeutics.

VE800: an orally administered live biotherapeutic product consisting of 11 distinct nonpathogenic, nontoxigenic, commensal bacterial strains manufactured under GMP conditions. 


\section{CONCLUSIONS AND FUTURE DIRECTIONS}

Both of the gut microbiota and the immune system are quite complex systems in our body, the currently proven relationship between them is only the tip of the iceberg. According to the existing research reports, the gut microbiota has a considerable relationship with the effect of tumor immunotherapy. We have summarized the abundance and composition changes of gut microbiota during immunotherapy of ICIs. Most of these bacteria are associated with immune system especially $\mathrm{T}$ cells. Some bacteria can significantly enhance the antitumor effect of ICIs, which may give us a hint that we can use gut microbiota as an aid to immunotherapy. In addition, specific intestinal bacteria can be used as a biomarker for immunotherapy. Although most of the mechanisms of antitumor effect of bacteria remain unknown, so the next step might be exploring the inner mechanism of gut microbiota and checkpoint inhibitor immunotherapy.

There still exist some limitations of previous research, we know that gut microbiota is a homeostatic ecosystem. Although regulating one certain flora can regulate the efficacy of ICIs immunotherapy, it also causes unbalance of the whole system, which might have some potential risks to human body. At present, the classification of intestinal flora is mostly done by 16s rRNA sequencing. 16s rRNA is highly conserved in many different kinds of bacteria, so the classification of flora based on 16s rRNA sequencing is relatively inaccurate. What's more, present analysis was mainly based on the fecal sample, but the microbes in the fecal

\section{REFERENCES}

1. Qin J, Li R, Raes J, Arumugam M, Burgdorf KS, Manichanh C, et al. A Human Gut Microbial Gene Catalogue Established by Metagenomic Sequencing. Nature (2010) 464(7285):59-65. doi: 10. 1038 /nature 08821

2. Cho I, Blaser MJ. The Human Microbiome: At the Interface of Health and Disease. Nat Rev Genet (2012) 13(4):260-70. doi: 10.1038/nrg3182

3. Renz H, Brandtzaeg P, Hornef $M$. The Impact of Perinatal Immune Development on Mucosal Homeostasis and Chronic Inflammation. Nat Rev Immunol (2011) 12(1):9-23. doi: 10.1038/nri3112

4. Human Microbiome Project C. Structure, Function and Diversity of the Healthy Human Microbiome. Nature (2012) 486(7402):207-14. doi: 10.1038/ nature 11234

5. Virtue AT, McCright SJ, Wright JM, Jimenez MT, Mowel WK, Kotzin JJ, et al. The Gut Microbiota Regulates White Adipose Tissue Inflammation and Obesity Via a Family of MicroRNAS. Sci Transl Med (2019) 11(496): eaav1892. doi: 10.1126/scitranslmed.aav1892

6. Livanos AE, Greiner TU, Vangay P, Pathmasiri W, Stewart D, McRitchie S, et al. Antibiotic-Mediated Gut Microbiome Perturbation Accelerates Development of Type 1 Diabetes in Mice. Nat Microbiol (2016) 1 (11):16140. doi: 10.1038/nmicrobiol.2016.140

7. Maini Rekdal V, Bess EN, Bisanz JE, Turnbaugh PJ, Balskus EP. Discovery and Inhibition of an Interspecies Gut Bacterial Pathway for Levodopa Metabolism. Science (2019) 364(6445):eaau6323. doi: 10.1126/ science.aau6323

8. Jin C, Lagoudas GK, Zhao C, Bullman S, Bhutkar A, Hu B, et al. Commensal Microbiota Promote Lung Cancer Development Via Gammadelta T Cells. Cell (2019) 176(5):998-1013 e16. doi: 10.1016/j.cell.2018.12.040

9. Tilg H, Adolph TE, Gerner RR, Moschen AR. The Intestinal Microbiota in Colorectal Cancer. Cancer Cell (2018) 33(6):954-64. doi: 10.1016/ j.ccell.2018.03.004 sample do not completely reflect the real ecosystem inside the intestine, so how to detect the dynamic change of gut microbiota after ICIs treatment in the human body remains a problem.

In conclusion, linking intestinal flora with tumor immunotherapy is a new attempt, and based on the existing research, the gut microbiota has a significant impact on the effect of tumor immunotherapy. Which prove that the various systems of the human body influence each other.

\section{AUTHOR CONTRIBUTIONS}

$\mathrm{HW}$ and LC are directors of this paper. HW is responsible for the main direction of this paper. LC designed the structure of this paper. JW was responsible for writing of major part of this paper. SW was responsible for proofreading of this paper. BZ was responsible for writing the abstract of this paper. XQ was responsible for writing the introduction of this paper. All authors contributed to the article and approved the submitted version.

\section{FUNDING}

This work was supported by the National Research Program of China (2017YFA0505803, 2017YFC0908100), the State Key project for Infectious Diseases (2018ZX10732202001,2018ZX10302207-004), and National Natural Science Foundation of China (81790633, 61922047, and 81902412).

10. Zitvogel L, Daillere R, Roberti MP, Routy B, Kroemer G. Anticancer Effects of the Microbiome and Its Products. Nat Rev Microbiol (2017) 15(8):465-78. doi: $10.1038 /$ nrmicro.2017.44

11. Garrett WS. Cancer and the Microbiota. Science (2015) 348(6230):80-6. doi: 10.1126/science.aaa4972

12. Shi T, Ma Y, Yu L, Jiang J, Shen S, Hou Y, et al. Cancer Immunotherapy: A Focus on the Regulation of Immune Checkpoints. Int J Mol Sci (2018) 19 (5):1389. doi: 10.3390/ijms19051389

13. Ribas A, Wolchok JD. Cancer Immunotherapy Using Checkpoint Blockade. Science (2018) 359(6382):1350-5. doi: 10.1126/science.aar4060

14. Wolchok JD. Pd-1 Blockers. Cell (2015) 162(5):937. doi: 10.1016/ j.cell.2015.07.045

15. Topalian SL, Hodi FS, Brahmer JR, Gettinger SN, Smith DC, McDermott DF, et al. Safety, Activity, and Immune Correlates of Anti-PD-1 Antibody in Cancer. N Engl J Med (2012) 366(26):2443-54. doi: 10.1056/NEJMoa1200690

16. Frelaut M, Le Tourneau C, Borcoman E. Hyperprogression Under Immunotherapy. Int J Mol Sci (2019) 20(11):2674. doi: 10.3390/ijms20112674

17. Gopalakrishnan V, Spencer CN, Nezi L, Reuben A, Andrews MC, Karpinets TV, et al. Gut Microbiome Modulates Response to Anti-PD-1 Immunotherapy in Melanoma Patients. Science (2018) 359(6371):97-103. doi: 10.1126/ science.aan 4236

18. Sivan A, Corrales L, Hubert N, Williams JB, Aquino-Michaels K, Earley ZM, et al. Commensal Bifidobacterium Promotes Antitumor Immunity and Facilitates Anti-PD-L1 Efficacy. Science (2015) 350(6264):1084-9. doi: 10.1126/science.aac4255

19. Vetizou M, Pitt JM, Daillere R, Lepage P, Waldschmitt N, Flament C, et al. Anticancer Immunotherapy by CTLA-4 Blockade Relies on the Gut Microbiota. Science (2015) 350(6264):1079-84. doi: 10.1126/science.aad1329

20. Routy B, Le Chatelier E, Derosa L, Duong CPM, Alou MT, Daillere R, et al. Gut Microbiome Influences Efficacy of PD-1-based Immunotherapy Against Epithelial Tumors. Science (2018) 359(6371):91-7. doi: 10.1126/ science.aan3706 
21. Thaiss CA, Zmora N, Levy M, Elinav E. The Microbiome and Innate Immunity. Nature (2016) 535(7610):65-74. doi: 10.1038/nature18847

22. Gopalakrishnan V, Helmink BA, Spencer CN, Reuben A, Wargo JA. The Influence of the Gut Microbiome on Cancer, Immunity, and Cancer Immunotherapy. Cancer Cell (2018) 33(4):570-80. doi: 10.1016/ j.ccell.2018.03.015

23. Johansson ME, Jakobsson HE, Holmen-Larsson J, Schutte A, Ermund A, Rodriguez-Pineiro AM, et al. Normalization of Host Intestinal Mucus Layers Requires Long-Term Microbial Colonization. Cell Host Microbe (2015) 18 (5):582-92. doi: 10.1016/j.chom.2015.10.007

24. Okai S, Usui F, Yokota S, Hori IY, Hasegawa M, Nakamura T, et al. HighAffinity Monoclonal IgA Regulates Gut Microbiota and Prevents Colitis in Mice. Nat Microbiol (2016) 1(9):16103. doi: 10.1038/nmicrobiol.2016.103

25. Honda K, Littman DR. The Microbiota in Adaptive Immune Homeostasis and Disease. Nature (2016) 535(7610):75-84. doi: 10.1038/nature18848

26. Donaldson GP, Ladinsky MS, Yu KB, Sanders JG, Yoo BB, Chou WC, et al. Gut Microbiota Utilize Immunoglobulin A for Mucosal Colonization. Science (2018) 360(6390):795-800. doi: 10.1126/science.aaq0926

27. Ivanov II, Atarashi K, Manel N, Brodie EL, Shima T, Karaoz U, et al. Induction of Intestinal Th17 Cells by Segmented Filamentous Bacteria. Cell (2009) 139 (3):485-98. doi: 10.1016/j.cell.2009.09.033

28. Atarashi K, Tanoue T, Shima T, Imaoka A, Kuwahara T, Momose Y, et al. Induction of Colonic Regulatory T Cells by Indigenous Clostridium Species. Science (2011) 331(6015):337-41. doi: 10.1126/science.1198469

29. Atarashi K, Tanoue T, Oshima K, Suda W, Nagano Y, Nishikawa H, et al. Treg Induction by a Rationally Selected Mixture of Clostridia Strains From the Human Microbiota. Nature (2013) 500(7461):232-6. doi: 10.1038/ nature12331

30. Parada Venegas D, de la Fuente MK, Landskron G, Gonzalez MJ, Quera R, Dijkstra G, et al. Short Chain Fatty Acids (Scfas)-Mediated Gut Epithelial and Immune Regulation and Its Relevance for Inflammatory Bowel Diseases. Front Immunol (2019) 10:277. doi: 10.3389/fimmu.2019.00277

31. Yoo JY, Groer M, Dutra SVO, Sarkar A, McSkimming DI. Gut Microbiota and Immune System Interactions. Microorganisms (2020) 8(10):1587. doi: $10.3390 /$ microorganisms 8101587

32. Furusawa Y, Obata Y, Fukuda S, Endo TA, Nakato G, Takahashi D, et al. Commensal Microbe-Derived Butyrate Induces the Differentiation of Colonic Regulatory T Cells. Nature (2013) 504(7480):446-50. doi: 10.1038/ nature 12721

33. Arpaia N, Campbell C, Fan X, Dikiy S, van der Veeken J, deRoos P, et al. Metabolites Produced by Commensal Bacteria Promote Peripheral Regulatory T-cell Generation. Nature (2013) 504(7480):451-5. doi: 10.1038/nature12726

34. Singh N, Gurav A, Sivaprakasam S, Brady E, Padia R, Shi H, et al. Activation of Gpr109a, Receptor for Niacin and the Commensal Metabolite Butyrate, Suppresses Colonic Inflammation and Carcinogenesis. Immunity (2014) 40 (1):128-39. doi: 10.1016/j.immuni.2013.12.007

35. Konieczna P, Ferstl R, Ziegler M, Frei R, Nehrbass D, Lauener RP, et al. Immunomodulation by Bifidobacterium Infantis 35624 in the Murine Lamina Propria Requires Retinoic Acid-Dependent and Independent Mechanisms. PloS One (2013) 8(5):e62617. doi: 10.1371/journal.pone.0062617

36. Round JL, Mazmanian SK. Inducible Foxp3+ Regulatory T-cell Development by a Commensal Bacterium of the Intestinal Microbiota. Proc Natl Acad Sci USA (2010) 107(27):12204-9. doi: 10.1073/pnas.0909122107

37. Round JL, Lee SM, Li J, Tran G, Jabri B, Chatila TA, et al. The Toll-like Receptor 2 Pathway Establishes Colonization by a Commensal of the Human Microbiota. Science (2011) 332(6032):974-7. doi: 10.1126/science.1206095

38. Polk DB, Peek RMJr. Helicobacter Pylori: Gastric Cancer and Beyond. Nat Rev Cancer (2010) 10(6):403-14. doi: 10.1038/nrc2857

39. Garrett WS. The Gut Microbiota and Colon Cancer. Science (2019) 364 (6446):1133-5. doi: 10.1126/science.aaw2367

40. Wong BC, Lam SK, Wong WM, Chen JS, Zheng TT, Feng RE, et al. Helicobacter Pylori Eradication to Prevent Gastric Cancer in a High-Risk Region of China: A Randomized Controlled Trial. JAMA (2004) 291(2):18794. doi: 10.1001/jama.291.2.187

41. Dai Z, Coker OO, Nakatsu G, Wu WKK, Zhao L, Chen Z, et al. Multi-Cohort Analysis of Colorectal Cancer Metagenome Identified Altered Bacteria Across Populations and Universal Bacterial Markers. Microbiome (2018) 6(1):70. doi: 10.1186/s40168-018-0451-2
42. Wong SH, Zhao L, Zhang X, Nakatsu G, Han J, Xu W, et al. Gavage of Fecal Samples From Patients With Colorectal Cancer Promotes Intestinal Carcinogenesis in Germ-Free and Conventional Mice. Gastroenterology (2017) 153(6):1621-33 e6. doi: 10.1053/j.gastro.2017.08.022

43. Wu S, Rhee KJ, Albesiano E, Rabizadeh S, Wu X, Yen HR, et al. A Human Colonic Commensal Promotes Colon Tumorigenesis Via Activation of T Helper Type 17 T Cell Responses. Nat Med (2009) 15(9):1016-22. doi: 10.1038/nm.2015

44. Pleguezuelos-Manzano C, Puschhof J, Rosendahl Huber A, van Hoeck A, Wood HM, Nomburg J, et al. Mutational Signature in Colorectal Cancer Caused by Genotoxic Pks(+) E. Coli. Nature (2020) 580(7802):269-73. doi: 10.1038/s41586-020-2080-8

45. Dapito DH, Mencin A, Gwak GY, Pradere JP, Jang MK, Mederacke I, et al. Promotion of Hepatocellular Carcinoma by the Intestinal Microbiota and TLR4. Cancer Cell (2012) 21(4):504-16. doi: 10.1016/j.ccr.2012.02.007

46. Yoshimoto S, Loo TM, Atarashi K, Kanda H, Sato S, Oyadomari S, et al. Obesity-Induced Gut Microbial Metabolite Promotes Liver Cancer Through Senescence Secretome. Nature (2013) 499(7456):97-101. doi: 10.1038/ nature 12347

47. Schwabe RF, Seki E, Brenner DA. Toll-Like Receptor Signaling in the Liver. Gastroenterology (2006) 130(6):1886-900. doi: 10.1053/j.gastro.2006.01.038

48. Pradere JP, Troeger JS, Dapito DH, Mencin AA, Schwabe RF. Toll-Like Receptor 4 and Hepatic Fibrogenesis. Semin Liver Dis (2010) 30(3):232-44. doi: 10.1055/s-0030-1255353

49. El-Serag HB. Hepatocellular Carcinoma. N Engl J Med (2011) 365(12):111827. doi: 10.1056/NEJMra1001683

50. Grat M, Wronka KM, Krasnodebski M, Masior L, Lewandowski Z, Kosinska I, et al. Profile of Gut Microbiota Associated With the Presence of Hepatocellular Cancer in Patients With Liver Cirrhosis. Transplant Proc (2016) 48(5):1687-91. doi: 10.1016/j.transproceed.2016.01.077

51. Schwabe RF, Greten TF. Gut Microbiome in HCC - Mechanisms, Diagnosis and Therapy. J hepatol (2020) 72(2):230-8. doi: 10.1016/j.jhep.2019.08.016

52. Chng KR, Chan SH, Ng AHQ, Li C, Jusakul A, Bertrand D, et al. Tissue Microbiome Profiling Identifies an Enrichment of Specific Enteric Bacteria in Opisthorchis Viverrini Associated Cholangiocarcinoma. EBioMedicine (2016) 8:195-202. doi: 10.1016/j.ebiom.2016.04.034

53. Bard J-M, Luu HT, Dravet F, Michel C, Moyon T, Pagniez A, et al. Relationship Between Intestinal Microbiota and Clinical Characteristics of Patients With Early Stage Breast Cancer. FASEB J (2015) 29:914.2. doi: 10.1096/fasebj.29.1_supplement.914.2

54. Luu TH, Michel C, Bard JM, Dravet F, Nazih H, Bobin-Dubigeon C. Intestinal Proportion of Blautia Sp. Is Associated With Clinical Stage and Histoprognostic Grade in Patients With Early-Stage Breast Cancer. Nutr Cancer (2017) 69(2):267-75. doi: 10.1080/01635581.2017.1263750

55. Dang AT, Marsland BJ. Microbes, Metabolites, and the Gut-Lung Axis. Mucosal Immunol (2019) 12(4):843-50. doi: 10.1038/s41385-019-0160-6

56. Zhou A, Lei Y, Tang L, Hu S, Yang M, Wu L, et al. Gut Microbiota: The Emerging Link to Lung Homeostasis and Disease. J Bacteriol (2020)203(4): e00454-20. doi: 10.1128/JB.00454-20

57. Grivennikov SI, Wang K, Mucida D, Stewart CA, Schnabl B, Jauch D, et al. Adenoma-Linked Barrier Defects and Microbial Products Drive IL-23/IL-17mediated Tumour Growth. Nature (2012) 491(7423):254-8. doi: 10.1038/ nature 11465

58. Kostic AD, Chun E, Meyerson M, Garrett WS. Microbes and Inflammation in Colorectal Cancer. Cancer Immunol Res (2013) 1(3):150-7. doi: 10.1158/23266066.CIR-13-0101

59. Hu Y, Le Leu RK, Christophersen CT, Somashekar R, Conlon MA, Meng XQ, et al. Manipulation of the Gut Microbiota Using Resistant Starch is Associated With Protection Against Colitis-Associated Colorectal Cancer in Rats. Carcinogenesis (2016) 37(4):366-75. doi: 10.1093/carcin/bgw019

60. Hu S, Dong TS, Dalal SR, Wu F, Bissonnette M, Kwon JH, et al. The MicrobeDerived Short Chain Fatty Acid Butyrate Targets miRNA-dependent p21 Gene Expression in Human Colon Cancer. PloS One (2011) 6(1):e16221. doi: 10.1371/journal.pone.0016221

61. Azcarate-Peril MA, Sikes M, Bruno-Barcena JM. The Intestinal Microbiota, Gastrointestinal Environment and Colorectal Cancer: A Putative Role for Probiotics in Prevention of Colorectal Cancer? Am J Physiol Gastrointest Liver Physiol (2011) 301(3):G401-24. doi: 10.1152/ajpgi.00110.2011 
62. Goodwin AC, Destefano Shields CE, Wu S, Huso DL, Wu X, Murray-Stewart TR, et al. Polyamine Catabolism Contributes to Enterotoxigenic Bacteroides Fragilis-Induced Colon Tumorigenesis. Proc Natl Acad Sci USA (2011) 108 (37):15354-9. doi: 10.1073/pnas.1010203108

63. Dossa AY, Escobar O, Golden J, Frey MR, Ford HR, Gayer CP. Bile Acids Regulate Intestinal Cell Proliferation by Modulating EGFR and FXR Signaling. Am J Physiol Gastrointest Liver Physiol (2016) 310(2):G81-92. doi: 10.1152/ ajpgi.00065.2015

64. Cao H, Luo S, Xu M, Zhang Y, Song S, Wang S, et al. The Secondary Bile Acid, Deoxycholate Accelerates Intestinal Adenoma-Adenocarcinoma Sequence in Apc (Min/+) Mice Through Enhancing Wnt Signaling. Fam Cancer (2014) 13 (4):563-71. doi: 10.1007/s10689-014-9742-3

65. Loo TM, Kamachi F, Watanabe Y, Yoshimoto S, Kanda H, Arai Y, et al. Gut Microbiota Promotes Obesity-Associated Liver Cancer Through PGE2Mediated Suppression of Antitumor Immunity. Cancer Discovery (2017) 7 (5):522-38. doi: 10.1158/2159-8290.CD-16-0932

66. Ma C, Han M, Heinrich B, Fu Q, Zhang Q, Sandhu M, et al. Gut MicrobiomeMediated Bile Acid Metabolism Regulates Liver Cancer Via NKT Cells. Science (2018) 360(6391):eaan5931. doi: 10.1126/science.aan5931

67. Allen-Vercoe E, Coburn B. A Microbiota-Derived Metabolite Augments Cancer Immunotherapy Responses in Mice. Cancer Cell (2020) 38(4):452-3 doi: 10.1016/j.ccell.2020.09.005

68. Mager LF, Burkhard R, Pett N, Cooke NCA, Brown K, Ramay H, et al. Microbiome-Derived Inosine Modulates Response to Checkpoint Inhibitor Immunotherapy. Science (2020) 369(6510):1481-9 doi: 10.1126/ science.abc3421

69. Peng Z, Cheng S, Kou Y, Wang Z, Jin R, Hu H, et al. The Gut Microbiome Is Associated With Clinical Response to Anti-PD-1/PD-L1 Immunotherapy in Gastrointestinal Cancer. Cancer Immunol Res (2020) 8(10):1251-61. doi: 10.1158/2326-6066.CIR-19-1014

70. Matson V, Fessler J, Bao R, Chongsuwat T, Zha Y, Alegre ML, et al. The Commensal Microbiome is Associated With Anti-PD-1 Efficacy in Metastatic Melanoma Patients. Sci (New York NY) (2018) 359(6371):104-8. doi: 10.1126/ science.aao 3290

71. Shi Y, Zheng W, Yang K, Harris KG, Ni K, Xue L, et al. Intratumoral Accumulation of Gut Microbiota Facilitates CD47-Based Immunotherapy Via STING Signaling. J Exp Med (2020) 217(5):e20192282. doi: 10.1084/ jem.20192282

72. Salgia NJ, Bergerot PG, Maia MC, Dizman N, Hsu J, Gillece JD, et al. Stool Microbiome Profiling of Patients With Metastatic Renal Cell Carcinoma Receiving Anti-Pd-1 Immune Checkpoint Inhibitors. Eur Urol (2020) 78 (4):498-502. doi: 10.1016/j.eururo.2020.07.011

73. Zheng $\mathrm{Y}$, Wang $\mathrm{T}$, Tu X, Huang $\mathrm{Y}$, Zhang $\mathrm{H}$, Tan D, et al. Gut Microbiome Affects the Response to anti-PD-1 Immunotherapy in Patients With Hepatocellular Carcinoma. J Immunother Cancer (2019) 7(1):193. doi: 10.1186/s40425-019-0650-9

74. Hakozaki T, Richard C, Elkrief A, Hosomi Y, Benlaifaoui M, Mimpen I, et al. The Gut Microbiome Associates With Immune Checkpoint Inhibition Outcomes in Patients With Advanced Non-Small Cell Lung Cancer. Cancer Immunol Res (2020) 8(10):1243-50. doi: 10.1158/2326-6066.CIR-20-0196

75. Derosa L, Routy B, Fidelle M, Iebba V, Alla L, Pasolli E, et al. Gut Bacteria Composition Drives Primary Resistance to Cancer Immunotherapy in Renal Cell Carcinoma Patients. Eur Urol (2020) 78(2):195-206. doi: 10.1016/ j.eururo.2020.04.044

76. Coley WB. The Classic - the Treatment of Malignant-Tumors by Repeated Inoculations of Erysipelas - With a Report of 10 Original Cases. Clin Orthop Relat $R$ (1991) 262):3-11. doi: 10.1097/00003086-199101000-00002
77. Chen ZF, Ai LY, Wang JL, Ren LL, Yu YN, Xu J, et al. Probiotics Clostridium Butyricum and Bacillus Subtilis Ameliorate Intestinal Tumorigenesis. Future Microbiol (2015) 10(9):1433-45. doi: 10.2217/fmb.15.66

78. Gianotti L, Morelli L, Galbiati F, Rocchetti S, Coppola S, Beneduce A, et al. A Randomized Double-Blind Trial on Perioperative Administration of Probiotics in Colorectal Cancer Patients. World J Gastroenterol (2010) 16 (2):167-75. doi: 10.3748/wjg.v16.i2.167

79. Pala V, Sieri S, Berrino F, Vineis P, Sacerdote C, Palli D, et al. Yogurt Consumption and Risk of Colorectal Cancer in the Italian European Prospective Investigation Into Cancer and Nutrition Cohort. Int $J$ Cancer (2011) 129(11):2712-9. doi: 10.1002/ijc.26193

80. Janket SJ, Ackerson LK, Diamandis EP. Gut Microbiotas and Immune Checkpoint Inhibitor Therapy Response: A Causal or Coincidental Relationship? Clin Chem Lab Med (2019) 58(1):18-24. doi: 10.1515/cclm2019-0605

81. Suez J, Zmora N, Zilberman-Schapira G, Mor U, Dori-Bachash M, Bashiardes S, et al. Post-Antibiotic Gut Mucosal Microbiome Reconstitution Is Impaired by Probiotics and Improved by Autologous Fmt. Cell (2018) 174(6):1406-23. doi: 10.1016/j.cell.2018.08.047

82. Spencer CN, Gopalakrishnan V, McQuade J, Andrews MC, Helmink B, Khan MAW, et al. Abstract 2838: The Gut Microbiome (GM) and Immunotherapy Response Are Influenced by Host Lifestyle Factors. Cancer Res (2019) 79(13 Supplement):2838-. doi: 10.1158/1538-7445.Am2019-2838

83. David LA, Maurice CF, Carmody RN, Gootenberg DB, Button JE, Wolfe BE, et al. Diet Rapidly and Reproducibly Alters the Human Gut Microbiome. Nature (2014) 505(7484):559-63. doi: 10.1038/nature12820

84. Lee KA, Shaw HM, Bataille V, Nathan P, Spector TD. Role of the Gut Microbiome for Cancer Patients Receiving Immunotherapy: Dietary and Treatment Implications. Eur J Cancer (2020) 138:149-55. doi: 10.1016/ j.ejca.2020.07.026

85. Limburg PJ, Mahoney MR, Ziegler KL, Sontag SJ, Schoen RE, Benya R, et al. Randomized Phase II Trial of Sulindac, Atorvastatin, and Prebiotic Dietary Fiber for Colorectal Cancer Chemoprevention. Cancer Prev Res (Phila) (2011) 4(2):259-69. doi: 10.1158/1940-6207.CAPR-10-0215

86. Wang YH, Wiesnoski DH, Helmink BA, Gopalakrishnan V, Choi K, DuPont HL, et al. Fecal Microbiota Transplantation for Refractory Immune Checkpoint Inhibitor-Associated Colitis. Nat Med (2018) 24(12):1804-+. doi: 10.1038/s41591-018-0238-9

87. Davar D, Dzutsev AK, McCulloch JA, Rodrigues RR, Chauvin JM, Morrison RM, et al. Fecal Microbiota Transplant Overcomes Resistance to Anti-PD-1 Therapy in Melanoma Patients. Science (2021) 371(6529):595-602. doi: 10.1126/science.abf3363

88. Baruch EN, Youngster I, Ben-Betzalel G, Ortenberg R, Lahat A, Katz L, et al. Fecal Microbiota Transplant Promotes Response in ImmunotherapyRefractory Melanoma Patients. Science (2021) 371(6529):602-9. doi: $10.1126 /$ science.abb5920

Conflict of Interest: The authors declare that the research was conducted in the absence of any commercial or financial relationships that could be construed as a potential conflict of interest.

Copyright (c) 2021 Wu, Wang, Zheng, Qiu, Wang and Chen. This is an open-access article distributed under the terms of the Creative Commons Attribution License (CC BY). The use, distribution or reproduction in other forums is permitted, provided the original author(s) and the copyright owner(s) are credited and that the original publication in this journal is cited, in accordance with accepted academic practice. No use, distribution or reproduction is permitted which does not comply with these terms. 